Bull. Austral. Math. Soc:

VOL. $37(1988) \quad[411-414]$

\title{
AN INVERSION THEOREM \\ FOR SET-VALUED MAPS
}

\author{
D. AzE
}

\begin{abstract}
The aim of this paper is to give an inversion theorem for set-valued maps involving both some known results for functions and set-valued maps. To do this we introduce a notion of strict differentiability for set-valued maps and we use a Newton like method assuming the derivative to be surjective. Moreover we prove the pseudo-Lipschitz regularity of the inverse.
\end{abstract}

\section{INTRODUC'TION}

In this note, we prove an open mapping and inversion theorem for set-valued maps which involves the results of $[\mathbf{1 5}]$ and $[\mathbf{2 3}]$. To do this we introduce a notion of differentiability for set-valued maps similar to the one given in $[8]$ (see also $[\boldsymbol{6}],[\mathbf{1 3}],[\mathbf{1 0}]$, [20] and [22]). Some inversion theorems for multifunctions have already been proved in particular in $[3],[5],[\mathbf{1 1}]$ and also $[20],[23]$. The favourable situation occurs when the multifunction takes on its values in a finite dimensional space. When this is not the case the problem is slightly different. In the particular case of functions, it is proved (see [7], [12]) that the assumption of Halkin's Theorem [16] (surjectivity of the clerivative) is not sufficient to obtain the open mapping and local inversion theorem. In this case some uniformity is needed near the point where the inversion is studied. When working with set-valued maps, this uniformity is obtained by introducing a notion of strict differentiability and the inversion result is proved with the help of an iteration procedure of Newton type quite similar to the one used in the proof of the Ljusternik Graves Theorem $([\mathbf{1 5}],[\mathbf{1 8}]$ and also $[\mathbf{1}])$.

\section{Statement and PRoOf of the Result}

In the sequel $X, Y$ denote $B$ anach spaces with closed unit balls $B_{X}, B_{Y}$. For any non-void subsets $C, D$ of a metric space $(E, d)$, the Hausdorff excess of $C$ into $D$ is defined by $e(C, D)=\sup _{x \in C} d(x, D)$ where $d(x, D)=\inf _{y \in D} d(x, y)$. We also set $e(\emptyset, D)=0$ and $e(C, \emptyset)=+\infty$ if $C \neq \emptyset$. A multifunction or set-valued map $F: X \rightrightarrows Y$ is a subset of $X \times Y$. For $x \in X, F(x):=\{y \in Y ;(x, y) \in F\}$, the domain of $F$ is the set $\operatorname{dom} F=\{x \in X, F(x) \neq \emptyset\}$. A convex process is a multifunction $L: X \rightrightarrows Y$ such

Received 28 July 1987

Copyright Clearance Centre, Inc. Serial-fee code: 0004-9729/88 \$A2.00+0.00. 
that $L$ is a convex cone (see [24] for instance). The closed convex processes play the role of continuous linear maps in the theory of differentiability of multifunctions. A multifunction $F \rightrightarrows Y$ is said to be pseudo-Lipschitzian at $\left(x_{0}, y_{0}\right) \in F$ if there exists $\ell \geqslant 0$ and neighbourhoods $U$ of $x_{0}$ and $V$ of $y_{0}$ such that $U \subseteq \operatorname{dom} F$ and, for each $x_{1}, x_{2} \in U, e\left(F\left(x_{1}\right) \cap V, F\left(x_{2}\right)\right) \leqslant \ell\left\|x_{1}-x_{2}\right\|$.

DEFINITION. We say that the multifunction $F: X \rightrightarrows Y$ is strictly differentiable at $x_{0} \in \operatorname{dom} F$ if there exists a closed convex process $L: X \rightrightarrows Y$ such that, for each $\varepsilon>0$, there exist $r>0, \alpha>0$ such that

$$
\forall x \in x_{0}+r B_{X}, \forall u \in \alpha B_{X}, e\left(L(u) \cap B_{Y}+F(x), F(x+u)\right)<\varepsilon\|u\| .
$$

REMARK: The process $L$ is not unique since every subprocess $L^{\prime} \subseteq L$ (in particular $L^{\prime}=\{(0,0)\}$ also satisfies (1).

Examples: 1. A map $f: U \rightarrow Y, U \subseteq X$ open, which is strictly differentiable at $x_{0} \in U$ is also strictly differentiable as a multifunction at $x_{0}$. More generally,

2. Let $f: U \rightarrow Y$ be strictly differentiable at $x_{0}$. If we define $F(x)=$ $f(x)+K$ where $K$ is a convex closed cone, then $F$ is strictly differentiable at $x_{0}$ with $L(u)=f^{\prime}\left(x_{0}\right)(u)+K$. Indeed $e\left(f^{\prime}\left(x_{0}\right)(u)+K+f(x)+K, f(x+u)+K\right) \leqslant$ $\left\|f(x+u)-f(x)-f^{\prime}\left(x_{0}\right)(u)\right\|$.

3. A closed convex process $L$ is strictly differentiable at every $x_{0} \in \operatorname{dom} L$. The following inversion theorem extends both Theorem 1 of [23] and Theorem 1 of [15].

ThEOREM. Assume that the multifunction $F$ is closed and strictly differentiable at $x_{0} \in \operatorname{dom} F$ and that $L$ is onto, then there exist $r>0, \eta>0$ such that

$$
F\left(x_{0}\right)+\eta B_{Y} \subseteq F\left(x_{0}+r B_{X}\right) .
$$

Moreover, $F^{-1}$ is pseudo-Lipschitz near $\left(y_{0}, x_{0}\right)$ for each $y_{0} \in F\left(x_{0}\right)$ that is there exist $\eta_{0}, r_{0}, \ell_{0}>0$ such that $\forall y_{1}, y_{2} \in y_{0}+\eta_{0} B_{Y}$

$$
e\left(F^{-1}\left(y_{1}\right) \cap\left(x_{0}+r_{0} B_{X}\right), F^{-1}\left(y_{2}\right) \cap\left(x_{0}+2 r_{0} B_{X}\right)\right) \leqslant \ell_{0}\left\|y_{1}-y_{2}\right\|
$$

Proof: We follow the lines of [1, Theorem 2.1]. As $L$ is onto, there exists (see [4, Corollary 3 p. 132]) $\gamma>0$ such that $B_{Y} \subseteq L\left(\gamma B_{X}\right)$. Thus, for each $v \in Y$, there exists $u \in X$ with $v \in L(u)$ and $\|u\| \leqslant \gamma\|v\|$, we write $u=L^{-1}(v)$. Consider now $0<\varepsilon<1$ and $r, \alpha$ associated with $\frac{\varepsilon}{\gamma}$ by (1). Let us choose $\eta>0$ such that $\eta<\inf \left(1, \frac{\alpha}{\gamma}, \frac{r(1-e)}{\gamma}\right)$. Using (1) we derive that

$$
\forall v \in \eta B_{Y}, \forall x \in x_{0}+r B_{X}, e\left(v+F(x), F\left(x+L^{-1}(v)\right)\right)<\varepsilon\|v\| .
$$


Let us consider $y_{0} \in F\left(x_{0}\right)$ and $\bar{y} \in y_{0}+\eta B_{Y}$. We set $x_{-1}=x_{0}$ and we assume that, for $n \in \mathbb{N}$ and $i \in[0, n]$

(5) $x_{i} \in x_{0}+r B_{X}, y_{i} \in F\left(x_{i}\right),\left\|x_{i}-x_{i-1}\right\| \leqslant \gamma\left\|\bar{y}-y_{0}\right\| \varepsilon^{i-1},\left\|y_{i}-\bar{y}\right\| \leqslant \varepsilon^{i}\left\|y_{0}-\bar{y}\right\|$.

Replacing in (4) $v$ by $\bar{y}-y_{n}, x$ by $x_{n}$ and using the fact that $y_{n} \in F\left(x_{n}\right)$ we obtain $d\left(\bar{y}, F\left(x_{n}+L^{-1}\left(\bar{y}-y_{n}\right)\right)\right)<\varepsilon\left\|\bar{y}-y_{n}\right\|$. Thus there exists $y_{n+1} \in$ $F\left(x_{n}+L^{-1}\left(\bar{y}-y_{n}\right)\right)$ such that $\left\|y_{n+1}-\bar{y}\right\| \leqslant \varepsilon\left\|y_{n}-\bar{y}\right\|$. Let us set $x_{n+1}=x_{n}+$ $L^{-1}\left(\bar{y}-y_{n}\right)$, then $\left\|x_{n+1}-x_{n}\right\| \leqslant \gamma\left\|\bar{y}-y_{n}\right\| \leqslant \gamma\left\|\bar{y}-y_{0}\right\| \varepsilon^{n}$. Hence (5) holds for $n+1$. The sequences $\left(x_{n}\right)$ and $\left(y_{n}\right)$ converge respectively to $\bar{x} \in x_{0}+\frac{\gamma\left\|\bar{y}-y_{0}\right\|}{1-\varepsilon} B_{X} \subseteq x_{0}+r B_{X}$ and to $\bar{y}$. As $y_{n} \in F\left(x_{n}\right)$ and $F$ is closed, one has $\bar{y} \in F(\bar{x})$ and (2) holds.

\section{Lipschitz dependence.}

Let us assume now that $\eta<\inf \left(1, \frac{\alpha}{\gamma}, \frac{r(1-\varepsilon)}{2 \gamma}\right)$. Thanks to the first step of the proof, there exists, for $y_{1} \in y_{0}+\frac{\eta}{2} B_{Y}$ an element $x_{1} \in x_{0}+\frac{r}{2} B_{X}$ such that $y_{1} \in$ $F\left(x_{1}\right)$. Consider $y_{2} \in y_{0}+\frac{\eta}{2} B_{Y}$, one has $y_{2} \in y_{1}+\eta B_{Y}, y_{1} \in F\left(x_{1}\right)$ and, by (4) $\forall v \in \eta B_{Y}, \forall x \in x_{1}+\frac{r}{2} B_{X}, e\left(v+F(x), F\left(x+L^{-1}(v)\right)\right)<\varepsilon\|v\|$. Hence, by using the same device as in the first step, there exists $x_{2} \in X$ such that $y_{2} \in F\left(x_{2}\right)$ and $\left\|x_{2}-x_{1}\right\| \leqslant \frac{\gamma}{\sigma^{\varepsilon}}\left\|y_{2}-y_{1}\right\|$, then (3) holds with $\eta_{0}=\frac{\eta}{2}, r_{0}=\frac{r}{2}, \ell_{0}=\frac{\gamma}{1-\varepsilon}$.

\section{REFERENCES}

[1] M. Altman, 'Inverse differentiability, contractors and equations in Banach space', Studia Math. 46 (1973), 1-15.

[2] M. Altman, 'Directional contractors and equations in Banach spaces', Studia Math. 46 (1973), 101-110.

[3] J-P. Aubin, 'Contingent derivative of set-valued maps and existence of solutions to nonlinear inclusions and differential inclusions', Mathematical analysis and applications, Part $A$ (Advances in Math. Suppl. Stud. 7A) (1981), 159-229.

[4] J-P. Aubin and I. Ekeland, Applied nonlinear analysis (J. Wiley, 1984).

[5] J-P. Aubin and H. Frankowska, 'On inverse function theorems for set-valued maps', J. Math. Pures Appl. 60 (1987), 71-89.

[0] H.T. Banks and M.Q. Jacobs, 'A differential calculus for multifunctions', J. Math. Anal. Appl. 29 (1970), 246-272.

[7] W. Barit and G.R. Wood, 'Differentiable retracts and a modified inverse function theorem', Bull. Austral. Math. Soc. 18 (1978), 37-43.

[8] F.S. De Blasi, 'On the differentiability of multifunctions', Pacific J. Math. 68 (1976), 67-81.

[9] S.N. Chow and A. Lasota, 'An implicit function theorem for nondifferentiable mappings', Proc. Amer. Math. Soc. 34 (1972), 141-146.

[10] F.H. Clarke, 'On the inverse function theorem', Pacific. J. Math. 64 (1976), 97-102.

[11] H. Frankowska, 'Théorème d'application ouverte pour les correspondances', C.R. Acad. Sci. Paris. Sér I Math. 302 (1986), 559-568.

[12] B. M. Garay, 'On an inverse theorem of Halkin', Annales Univ. Sci. Budapest 38 (1982), 129-131.

[13] S. Gautier, Différentiabilité des multiapplication, (Publications Mathématiques Université de Pau), 1978. 
[14] S. Gautier and G. Isac and J-P. Penot, 'Surjectivity of multifunctions under generalised differentiability assumptions', Bull. Austral. Math. Soc. 28 (1983), 13-21.

[15] L.M. Graves, 'Some mapping theorems', Duke Math. 17 (1950), 111-114.

[16] H. Halkin, 'Implicit functions and optimization problems without continuous differentiability of the data', $S I A M J$. Control 12 (1974), 229-236.

[17] H. Halkin, 'Interior mapping theorem with set-valued derivatives', Jour. Analyse Math. 30 (1976), 200-207.

[18] L.A. Ljusternik and S.L. Sobolev, Elements of functional analysis (Ungar New-York, 1961).

[10] M. Martelli and A. Vignoli, 'On difterentiability of multivalued maps', Boll. Un. Mat. Ital. (4) 10 (1974), 701-712.

[20] H. Methlouti, Cahiers du CEREMADE n० 7702, in, Univ. Paris IX, 1977.

[21] S. Mirica, 'A note on the generalised differentiability of mappings', Nonlinear Anal. 4 (1980), $567-575$.

[22] E.A. Nurminski, 'On differentiability of multifunctions', (Russian), Kibernetika (Kiev) 5 (1978), 46-48.

[23] S.M. Robinson, 'An inverse function theorem for a class of multivalued functions', Proc. Amer. Math. Soc. 41, 1 (1973), 211-218.

[24] S.M. Robinson, 'Normed convex processes', Trans. Amer. Math. Soc. 174 (1972), $127-140$.

[25] S.M. Robinson, 'Regularity and stability for convex multivalued function', Math. Oper. Res. 1 (1976), 130-143.

[20] C. Urcescu, 'Multifunctions with convex closed graplis', (100), Czechoslovak Math. J. 25 (1975), 428-441.

\author{
Université de Perpignan \\ Mathématiques \\ Av, de Villeneuve \\ 66025 Perpignan, Cedex \\ France
}

